Invent. math. 126, 205-214 (1996)

Inventiones

matbematicae

(C) Springer-Verlag 1996

\title{
Algebraic limits of Kleinian groups which rearrange the pages of a book
}

\author{
James W. Anderson ${ }^{1, \star}$, Richard D. Canary ${ }^{2, \star \star}$ \\ ${ }^{1}$ Department of Mathematics, Rice University, Houston, TX 77251, USA \\ ${ }^{2}$ Department of Mathematics, University of Michigan, Ann Arbor, MI 48109, USA
}

Oblatum 10-IV-1995 \& 14-III-1996

Dedicated to Bernard Maskit on the occasion of his sixtieth birthday

\section{Introduction}

In this paper, we give examples of two new phenomena in Kleinian groups. We first exhibit a sequence of homeomorphic marked hyperbolic 3-manifolds whose algebraic limit is not homeomorphic to any element in the sequence. We then use this construction to exhibit situations where the space of convex co-compact representations of a given 3-manifold group has many components but its closure is connected.

Let $M$ be a compact, irreducible, oriented 3-manifold and let $\mathscr{D}\left(\pi_{1}(M)\right)$ denote the space of all discrete, faithful representations of $\pi_{1}(M)$ into $\operatorname{PSL}_{2}(\mathbf{C})$. A sequence of representations $\left\{\rho_{n}\right\} \subset \mathscr{D}\left(\pi_{1}(M)\right)$ converging to $\rho \in \mathscr{D}\left(\pi_{1}(M)\right)$ gives rise to a sequence $\left\{N_{\rho_{n}}=\mathbf{H}^{3} / \rho_{n}\left(\pi_{1}(M)\right)\right\}$ of hyperbolic 3-manifolds, each of which is homotopy equivalent to $M$. The sequence $\left\{N_{\rho_{n}}\right\}$ is said to converge algebraically to $N_{\rho}=\mathbf{H}^{3} / \rho\left(\pi_{1}(M)\right.$ ). (See $[7,13,14]$ for more information about algebraic convergence of Kleinian groups.) In many situations (see $[1,6,15,24,25,27]$ ), it has been shown that $N_{\rho_{n}}$ must be homeomorphic to $N_{\rho}$ for all large enough $n$, and we had suspected that this would always be the case. In this paper, we give a collection of examples where $N_{\rho_{n}}$ is not homeomorphic to $N_{\rho}$ for any $n$. Our sequences are quite well-behaved: the $\rho_{n}\left(\pi_{1}(M)\right)$ are convex co-compact and mutually quasiconformally conjugate, and the algebraic limit $\rho\left(\pi_{1}(M)\right)$ is geometrically finite.

In our examples, $M$ is obtained by gluing a collection of $I$-bundles to a solid torus along a family of parallel annuli. These manifolds are particularly simple examples of books of $I$-bundles (see [9]) where, to explain the terminology, one should think of the solid torus as the binding and the $I$-bundles

\footnotetext{
${ }^{\star}$ Current address: Faculty of Mathematical Studies, University of Southampton Highfield, Southampton SO17 1BJ, England.

$\star \star$ Partially supported by a grant from the National Science Foundation and a fellowship from the Alfred P. Sloan Foundation.
} 
as the pages. The main tool in our construction is a version of Thurston's hyperbolic Dehn surgery theorem which is due to Tim Comar [8] (see also Bonahon-Otal [3]). We use this theorem to produce a sequence $\left\{\rho_{n}^{\prime}\right\}$ of convex co-compact uniformizations of $M$ converging to $\rho^{\prime} \in \mathscr{D}\left(\pi_{1}(M)\right)$ such that $N_{\rho^{\prime}}$ is homeomorphic to the interior of $M$, and $\left\{N_{\rho_{n}}\right\}$ converges "geometrically" to a geometrically finite hyperbolic 3-manifold $\widehat{N}$ which is homeomorphic to the interior of $M-\delta$, where $\delta$ is the core curve of the solid torus. (This type of phenomenon was first discovered by Jørgensen $[12,15]$ and was subsequently investigated by Marden [18], Thurston [27], and others, e.g. see [3, 8, 16, 24, and 28].) If $M^{\tau}$ is homotopy equivalent to one of our examples, then it is also obtained by gluing the same collection of $I$-bundles to a solid torus along the same family of parallel annuli, although perhaps in a different order. In particular, there is a cover $N^{\tau}$ of $\widehat{N}$ which is homeomorphic to the interior of $M^{\tau}$. We will see that one may precompose the representations in the sequence $\left\{\rho_{n}^{\prime}\right\}$ by a sequence $\left\{\chi_{n}\right\}$ of automorphisms of $\pi_{1}(M)$ so that the resulting sequence of representations $\left\{\rho_{n}=\rho_{n}^{\prime} \circ \chi_{n}\right\}$ converges to a representation $\rho \in \mathscr{D}\left(\pi_{1}(M)\right)$ with $N_{\rho}=N^{\tau}$.

Our examples also serve to demonstrate new phenomena in the deformation theory of Kleinian groups. If we let $C C\left(\pi_{1}(M)\right)$ denote the set of convex cocompact representations of $\pi_{1}(M)$, the components of $C C\left(\pi_{1}(M)\right)$ are in a oneto-one correspondence with the marked homeomorphism types of irreducible (oriented) 3-manifolds homotopy equivalent to $M$. In our class of examples, the closure of $C C\left(\pi_{1}(M)\right)$ will be connected, although $C C\left(\pi_{1}(M)\right)$ can have arbitrarily many components. We note that there are other examples where the components of $C C\left(\pi_{1}(M)\right)$ are known to have disjoint closures. In a future paper [2] with Darryl McCullough, we will explore more general classes of examples.

The convex co-compact Kleinian groups correspond, via the Sullivan dictionary between rational maps and Kleinian groups (see [26]), to hyperbolic rational maps. Hence, this phenomenon is the analogue of hyperbolic components of the Mandelbrot set whose closures intersect. It is conjectured that $C C\left(\pi_{1}(M)\right)$ is dense in $\mathscr{D}\left(\pi_{1}(M)\right)$. This is analogous to the conjecture that the hyperbolic components are dense in the Mandelbrot set.

\section{The examples}

In this section, we construct the examples promised in the introduction. For the remainder of the paper, fix a positive integer $k \geqq 3$.

Let $V=D^{2} \times S^{1}$ be a solid torus and let $A(j)(1 \leqq j \leqq k)$ denote a family of $k$ disjoint parallel annuli in $\partial V$ such that the inclusion map of $A(j)$ into $V$ is a homotopy equivalence. (Explicitly, we could choose $A(j)=\left[e^{2 \pi i(4 j-1) / 4 k}\right.$, $\left.e^{2 \pi i(4 j+1) / 4 k}\right] \times S^{1}$.) Let $F(j)$ be a compact, oriented surface of genus $j$ with one boundary component. Let $B(j)=F(j) \times I$ and let $\partial_{0} B(j)=\partial F(j) \times I$. Form a manifold $M_{k}$ from $V$ and $\{B(1), \ldots, B(k)\}$ by identifying $\partial_{0} B(j)$ with $A(j)$ (by an orientation-reversing homeomorphism) for all $1 \leqq j \leqq k$. 
One may obtain a manifold which is homotopy equivalent to $M_{k}$, but is not homeomorphic to $M_{k}$, by simply rearranging the pages. More specifically, let $\tau$ be any permutation of $\{1, \ldots, k\}$, and form $M_{k}^{\tau}$ from $V$ and $\{B(1), \ldots, B(k)\}$ by identifying $\partial_{0}(B(\tau(j)))$ with $A(j)$. In the proof of Lemma 3.2, we will see that $M_{k}$ and $M_{k}^{\tau}$ are homeomorphic if and only if $\tau$ and $\tau^{\prime}$ are in the same right coset of the dihedral group $\mathbf{D}_{k}$ within the symmetric group $\mathbf{S}_{k}$. (Throughout the paper $\sigma \tau$ will denote the result of applying the permutation $\sigma$ and then $\tau$.)

A finitely generated, discrete subgroup $\Gamma$ of $\mathrm{PSL}_{2}(\mathbf{C})$ is convex co-compact (respectively geometrically finite) if the convex core $C(N)$ of $N=\mathbf{H}^{3} / \Gamma$ is compact (resp. finite volume). We say that $\Gamma$ uniformizes a compact 3-manifold $M$ if there exists an orientation-preserving homeomorphism between $N$ and the interior of $M$.

Explicit convex co-compact Kleinian groups realizing $M_{k}$ and $M_{k}^{\tau}$ (for any $\tau)$ can be constructed using the techniques of Klein-Maskit combination; see, for example, Maskit [19], particularly Chapter VIII.E. and Maskit [20]. In Remark 1, at the end of the section, we construct geometrically finite Kleinian groups uniformizing $M_{k}$ and $M_{k}^{\tau}$.

The properties of our main example are contained in the following theorem.

Theorem 2.1. Let $\tau$ be a permutation of $\{1, \ldots, k\}$. There exists a sequence $\left\{\rho_{n}\right\} \subset \mathscr{D}\left(\pi_{1}\left(M_{k}\right)\right)$ which converges algebraically to $\rho \in \mathscr{D}\left(\pi_{1}\left(M_{k}\right)\right)$ such that $\rho_{n}\left(\pi_{1}\left(M_{k}\right)\right)$ is convex co-compact and uniformizes $M_{k}$ for all $n$, and $\rho\left(\pi_{1}\left(M_{k}\right)\right)$ is geometrically finite and uniformizes $M_{k}^{\tau}$.

Proof of 2.1. We will use a construction outlined by Kerckhoff and Thurston [16] (and later generalized by Ohshika [24], Bonahon-Otal [3] and Comar [8]) which was originally used to produce a sequence of discrete, faithful representations of a surface group whose geometric limit properly contains its algebraic limit.

We first recall some of the notation of Dehn surgery. Let $\widehat{M}$ be a compact, irreducible, oriented 3-manifold whose boundary contains a single torus $T$; any other component of $\partial \widehat{M}$ has genus at least 2. Choose a meridian $m$ and longitude $l$ for the torus $T$, and think of $m$ and $l$ as a basis for $\pi_{1}(T)$. If $(p, q)$ is a pair of relatively prime integers, then $\widehat{M}(p, q)$ is the manifold obtained by attaching a solid torus $V$ to $\widehat{M}$ by an orientation-reversing homeomorphism which identifies the meridian of $V$ with a simple closed curve in the homotopy class of $m^{p} l^{q}$ on $T$.

We now state a version of Thurston's hyperbolic Dehn surgery theorem which is due, in this form, to Comar [8] (see also Bonahon-Otal [3]).

Theorem 2.2. ([8]). Let $\widehat{M}$ be a compact, oriented 3-manifold with one toroidal boundary component $T$. Let $\widehat{N}=\mathbf{H}^{3} / \widehat{\Gamma}$ be a geometrically finite hyperbolic 3-manifold and $\phi: \operatorname{int}(\widehat{M}) \rightarrow \widehat{N}$ an orientation-preserving homeomorphism between the interior of $\widehat{M}$ and $\widehat{N}$. Further assume that every parabolic element of $\widehat{\Gamma}$ is conjugate to an element of $\phi_{*}\left(\pi_{1}(T)\right)$. Let $\left\{\left(p_{n}, q_{n}\right)\right\}$ be a sequence of distinct pairs of relatively prime integers. 
Then, for all sufficiently large $n$, there exists a representation $\beta_{n}: \widehat{\Gamma} \rightarrow$ $\mathrm{PSL}_{2}(\mathbf{C})$ with discrete image such that

1. $\beta_{n}(\widehat{\Gamma})$ is convex co-compact and uniformizes $\widehat{M}\left(p_{n}, q_{n}\right)$,

2. the kernel of $\beta_{n}$ is normally generated by $m^{p_{n}} q^{q_{n}}$, and

3. $\left\{\beta_{n}\right\}$ converges to the identity representation of $\widehat{\Gamma}$.

Moreover, if we let $i_{n}$ denote the inclusion of $\widehat{M}$ into $\widehat{M}\left(p_{n}, q_{n}\right)$, then there exists an orientation-preserving homeomorphism $\phi_{n}: \operatorname{int}\left(\widehat{M}\left(p_{n}, q_{n}\right)\right)$ $\rightarrow \mathbf{H}^{3} / \beta_{n}(\widehat{\Gamma})$ such that $\beta_{n} \circ \phi_{*}$ is conjugate to $\left(\phi_{n}\right)_{*} \circ\left(i_{n}\right)_{*}$.

Recall the construction of $M_{k}$ given above. Form $\widehat{M}_{k}$ by attaching an annulus to $M_{k}$ along two parallel, homotopically non-trivial curves in $\partial V \cap \partial M_{k}$, and then thickening the annulus. We denote this additional thickened annulus by $R$. (Explicitly, let $C_{1}=\left\{e^{3 \pi i / 4 k}\right\} \times S^{1} \subset \partial V$ and let $C_{2}=\left\{e^{5 \pi i / 4 k}\right\} \times S^{1} \subset \partial V$ be two parallel curves in $\partial V \cap \partial M_{k}$. Form $\widehat{M}_{k}$ by attaching $S^{1} \times I \times I$ to $M_{k}$ by an embedding $h: S^{1} \times I \times\{0,1\} \rightarrow \partial V \cap \partial M_{k}$ such that $h\left(S^{1} \times\{1 / 2\} \times\{0\}\right)=$ $C_{1}$ and $h\left(S^{1} \times\{1 / 2\} \times\{1\}\right)=C_{2}$.) Notice that $\widehat{M}_{k}$ is homeomorphic to the manifold obtained by removing an open tubular neighborhood of the core curve of $V$ from $M_{k}$.

Let $T$ denote the unique toroidal boundary component of $\widehat{M}_{k}$. Choose a meridian $m$ and a longitude $l$ for $T$ so that $l$ is parallel to $C_{1}$. Let $i_{n}: \widehat{M}_{k} \rightarrow$ $\widehat{M}_{k}(1, n)$ and $f: M_{k} \rightarrow \widehat{M}_{k}$ denote inclusion maps. Note that for any integer $n \in \mathbf{Z}, \widehat{M}_{k}(1, n)$ is homeomorphic to $M_{k}$ and the inclusion $i_{n} \circ f: M_{k} \rightarrow$ $\widehat{M}_{k}(1, n)$ is a homotopy equivalence which is homotopic to an orientationpreserving homeomorphism.

One may check that Thurston's geometrization theorem (see [22]) guarantees that $\widehat{M}_{k}$ is uniformized by a geometrically finite Kleinian group $\widehat{\Gamma}_{k}$, such that every parabolic element of $\widehat{\Gamma}_{k}$ is conjugate to an element of $\pi_{1}(T)$. (We will later sketch, in Remark 1 at the end of the section, an explicit construction.) Let $\widehat{N}_{k}=\mathbf{H}^{3} / \widehat{\Gamma}_{k}$ and let $\phi: \operatorname{int}\left(\widehat{M}_{k}\right) \rightarrow \widehat{N}_{k}$ be an orientation-preserving homeomorphism.

Let $\left\{\beta_{n}: \widehat{\Gamma}_{k} \rightarrow \operatorname{PSL}_{2}(\mathbf{C})\right\}$ and $\left\{\phi_{n}: \operatorname{int}\left(\widehat{M}_{k}(1, n)\right) \rightarrow \mathbf{H}^{3} / \beta_{n}\left(\widehat{\Gamma}_{k}\right)\right\}$ be the sequences of representations and homeomorphisms produced by Theorem 2.2 for the sequence $\{(1, n)\}$. Set $\rho_{n}^{\prime}=\beta_{n} \circ \phi_{*} \circ f_{*}$. Since $\beta_{n} \circ \phi_{*} \circ f_{*}$ is conjugate to $\left(\phi_{n}\right)_{*} \circ\left(i_{n}\right)_{*} \circ f_{*}, \rho_{n}^{\prime}$ is faithful and has image $\beta_{n}\left(\widehat{\Gamma}_{k}\right)$. Thus, each $\rho_{n}^{\prime}\left(\pi_{1}\left(M_{k}\right)\right)$ is convex co-compact and uniformizes $M_{k}$. Moreover, $\left\{\rho_{n}^{\prime}\right\}$ converges to the representation $\rho^{\prime}=\phi_{*} \circ f_{*}$ with image $\phi_{*}\left(f_{*}\left(\pi_{1}\left(M_{k}\right)\right)\right)$. which uniformizes $M_{k}$.

In order to rearrange the pages, we first construct, given a permutation $\tau$ of $\{1, \ldots, k\}$, an immersion $f_{\tau}: M_{k}^{\tau} \rightarrow \widehat{M}_{k}$ such that

1. $\phi_{*}\left(\left(f_{\tau}\right)_{*}\left(\pi_{1}\left(M_{k}^{\tau}\right)\right)\right)$ is a geometrically finite uniformization of $M_{k}^{\tau}$, and

2. $\left(i_{n} \circ f_{\tau}\right)_{*}$ is an isomorphism for all $n$.

Having constructed such an $f_{\tau}$, we complete the proof by taking $\rho_{n}=$ $\beta_{n} \circ \phi_{*} \circ\left(f_{\tau}\right)_{*} \circ\left(h_{\tau}\right)_{*}$, where $h_{\tau}: M_{k} \rightarrow M_{k}^{\tau}$ is a homotopy equivalence which is the identity on the solid torus $V$. Since $\beta_{n} \circ \phi_{*} \circ\left(f_{\tau}\right)_{*}$ is conjugate to $\left(\phi_{n}\right)_{*} \circ\left(i_{n}\right)_{*} \circ\left(f_{\tau}\right)_{*}$, we see that $\rho_{n}$ is faithful and that $\rho_{n}\left(\pi_{1}\left(M_{k}\right)\right)=\beta_{n}\left(\widehat{\Gamma}_{k}\right)$. 
Hence, $\rho_{n}\left(\pi_{1}\left(M_{k}\right)\right)$ is a convex co-compact uniformization of $M_{k}$ for all $n$. However, this time $\left\{\rho_{n}\right\}$ converges to a representation $\rho=\phi_{*} \circ\left(f_{\tau}\right)_{*} \circ\left(h_{\tau}\right)_{*}$ of $\pi_{1}\left(M_{k}\right)$ with image $\phi_{*}\left(\left(f_{\tau}\right)_{*}\left(\pi_{1}\left(M_{k}^{\tau}\right)\right)\right)$ which is a geometrically finite uniformization of $M_{k}^{\tau}$.

The remainder of the proof consists of the construction of $f_{\tau}$. As this construction is the crux of the proof, we will give an alternative, more schematic, description of the immersion in Remark 2 at the end of the section. In Remark 1, we explicitly identify the subgroup $\phi_{*}\left(\left(f_{\tau}\right)_{*}\left(\pi_{1}\left(M_{k}^{\tau}\right)\right)\right)$ of $\widehat{\Gamma}_{k}$.

Let $H_{k}$ denote the subgroup of $\pi_{1}\left(\widehat{M}_{k}\right)$ which is normally generated by $\pi_{1}\left(M_{k}\right)$, and let $M_{k}^{\infty}$ be the cover of $\widehat{M}_{k}$ associated to $H_{k} . M_{k}^{\infty}$ consists of infinitely many homeomorphic lifts of $M_{k}$ joined together by infinitely many homeomorphic lifts of $R$. Let $\left(M_{k}\right)_{i}$ denote the $i^{t h}$ copy of $M_{k}$ and $B(j)_{i}$ the copy of $B(j)$ contained in $M_{i}$.

We construct $f_{\tau}: M_{k}^{\tau} \rightarrow \widehat{M}_{k}$ by first constructing $\tilde{f}_{\tau}: M_{k}^{\tau} \rightarrow M_{k}^{\infty}$ and then projecting. We first define $\tilde{f}_{\tau}$ on the pages of $M_{k}^{\tau}$. We let $\left.\tilde{f}_{\tau}\right|_{B(\tau(j))}$ be the natural identification of $B(\tau(j))$ with $B(\tau(j))_{j}$. We then extend $\tilde{f}_{\tau}$ to an embedding in such a way that $\tilde{f}_{\tau}(V)$ is contained entirely in lifts of $V$ and $R$.

In order to check property (1), we consider the cover $\widetilde{M}_{k}^{\tau}$ of $\operatorname{int}\left(\widehat{M}_{k}\right)$ associated to $\left(f_{\tau}\right)_{*}\left(\pi_{1}\left(M_{k}^{\tau}\right)\right)$. Since $\widetilde{M}_{k}^{\tau}$ covers $\widehat{M}_{k}^{\infty}, f_{\tau}$ lifts to an embedding $g_{\tau}: M_{k}^{\tau} \rightarrow \widetilde{M}_{k}^{\tau}$ which is a homotopy equivalence. Let $g_{\tau}^{\prime}: M_{k}^{\tau} \rightarrow \operatorname{int}\left(\widetilde{M}_{k}^{\tau}\right)$ be an embedding of $M_{k}^{\tau}$ into the interior of $\widetilde{M}_{k}^{\tau}$ which is homotopic to $g_{\tau}$. If $\Gamma_{k}^{\tau}=\phi_{*}\left(\left(f_{\tau}\right)_{*}\left(\pi_{1}\left(M_{k}^{\tau}\right)\right)\right)$ and $N_{k}^{\tau}=\mathbf{H}^{3} / \Gamma_{k}^{\tau}$, then $\phi$ lifts to a homeomorphism $\widetilde{\phi}: \operatorname{int}\left(\widetilde{M}_{k}^{\tau}\right) \rightarrow N_{k}^{\tau}$. Hence, $g_{\tau}^{\prime} \circ \widetilde{\phi}$ is an embedding of $M_{k}^{\tau}$ into $N_{k}^{\tau}$ which is a homotopy equivalence. $\Gamma_{k}^{\tau}$ is geometrically finite, as it is a finitely generated subgroup of a geometrically finite co-infinite volume Kleinian group (see Proposition 7.1 in [22]). Hence, $N_{k}^{\tau}$ is homeomorphic to the interior of a compact 3-manifold. However, since $N_{k}^{\tau}$ contains an embedded copy of $M_{k}^{\tau}$ whose inclusion map is a homotopy equivalence, we see that $N_{k}^{\tau}$ is homeomorphic to the interior of $M_{k}^{\tau}$ (see Theorem 1 in [21]). Thus property (1) holds.

We now check property (2). Fix a basepoint $*$ in $V$ and let $\alpha_{j}$ be a path joining $*$ to $A_{j}$ and lying entirely in $V$. Let $g$ be a generator of $\pi_{1}(V, *)$. Let $G_{j}$ denote $\pi_{1}\left(B(j) \cup \alpha_{j}, *\right)$ sitting as a subgroup of $\pi_{1}\left(M_{k}, *\right)$, and note that $\pi_{1}\left(M_{k}, *\right)$ is generated by $G_{1}, \ldots, G_{k}$. (Explicitly, the subgroup generated by $G_{1}, \ldots, G_{j}$ is the amalgamated free product of the subgroup generated by $G_{1}, \ldots, G_{j-1}$ and the subgroup $G_{j}$ amalgamated along the common cyclic subgroup generated by $g$.) Furthermore, $\pi_{1}\left(\widehat{M}_{k}, *\right)$ is generated by $\pi_{1}\left(M_{k}, *\right)$ and an element $h$ which commutes with $g$. If we let $G_{j}^{\tau}$ be the subgroup of $\pi_{1}\left(M_{k}^{\tau}, *\right)$ corresponding to $\pi_{1}\left(B(j) \cup \alpha_{\tau^{-1}(j)}, *\right)$, then $\pi_{1}\left(M_{k}^{\tau}, *\right)$ is generated by $G_{1}^{\tau}, \ldots, G_{k}^{\tau}$ (with a explicit description similar to that of $\pi_{1}\left(M_{k}, *\right)$ ). The restriction of $\left(f_{\tau}\right)_{*}$ to $G_{j}^{\tau}$ is an isomorphism onto $c_{j} G_{j} c_{j}^{-1}$, where $c_{j}$ is some element of $\langle g, h\rangle$. Thus, $\left(i_{n} \circ f_{\tau}\right)_{*}$ restricted to $G_{j}^{\tau}$ is an isomorphism onto $G_{j}$, since $\left(i_{n}\right)_{*}$ maps $c_{j}$ to some power of $g$ and $g$ normalizes $G_{j}$. One may then easily check that $\left(i_{n} \circ f_{\tau}\right)_{*}$ is an isomorphism. We have completed the proof. 
Remark 1. We now explain briefly how to construct $\widehat{\Gamma}_{k}$ using Klein-Maskit combination, and we identify the subgroups $\Gamma_{k}$ and $\Gamma_{k}^{\tau}$, in hopes of illuminating the construction.

Let $\xi_{a}(z)=z+a$ be the element of $\mathrm{PSL}_{2}(\mathbf{C})$ corresponding to translation by $a \in \mathbf{C}$. Let $\Theta_{j}$ be a subgroup of $\operatorname{PSL}_{2}(\mathbf{R})$ which uniformizes $F(j)$ and contains $\xi_{1}$ as a primitive element (which thus corresponds to the puncture of $F(j))$. Klein-Maskit combination theory [19] guarantees that we can choose $0=a_{1}<a_{2}<\cdots<a_{k}$ such that the group $\Gamma_{k}$ generated by $\xi_{a_{1} i} \Theta_{1} \xi_{a_{1} i}^{-1}, \ldots, \xi_{a_{k} i} \Theta_{k} \xi_{a_{k} i}^{-1}$ is a geometrically finite uniformization of $M_{k}$ such that every parabolic element of $\Gamma_{k}$ is conjugate to $\xi_{n}$ for some $n \in \mathbf{Z}$. Similarly, there exists $\mu>a_{k}$ such that the group $\widehat{\Gamma}_{k}$ generated by $\Gamma_{k}$ and $\xi_{\mu i}$ is a geometrically finite uniformization of $\widehat{M}_{k}$ such that every parabolic element is conjugate to an element of the subgroup $\left\langle\xi_{1}, \xi_{\mu i}\right\rangle$.

We can now identify $\Gamma_{k}^{\tau}$ quite explicitly. The meridian $m$ is identified with $\xi_{\mu i}$ and the longitude $l$ is identified with $\xi_{1}$. If we let $\Theta_{j}^{\prime}$ denote $\xi_{a_{j}} \Theta_{j} \xi_{a_{j}}^{-1}$, then $\Gamma_{k}^{\tau}$ is generated by

$$
\xi_{\mu i} \Theta_{\tau(1)}^{\prime} \xi_{\mu i}^{-1}, \xi_{2 \mu i} \Theta_{\tau(2)}^{\prime} \xi_{2 \mu i}^{-1}, \ldots, \xi_{k \mu i} \Theta_{\tau(k)}^{\prime} \xi_{k \mu i}^{-1}
$$

One can check directly, again using Klein-Maskit combination theory, that $\Gamma_{k}^{\tau}$ is a geometrically finite uniformization of $M_{k}^{\tau}$.

Remark 2. We now give a schematic description of $f_{\tau}$. Let $C_{1}, \ldots, C_{k}$ be a family of consecutive, parallel, disjoint simple closed curves on the annulus $A=S^{1} \times I$, where $C_{1}=S^{1} \times\{0\}$ and $C_{k}=S^{1} \times\{1\}$. Let $X_{k}^{\tau}$ be the 2complex obtained from $A$ and $\{F(1), \ldots, F(k)\}$ by identifying $\partial F(\tau(j))$ with $C_{j}$. The 3-manifold $M_{k}^{\tau}$ is a thickening of the 2-complex $X_{k}^{\tau}$.

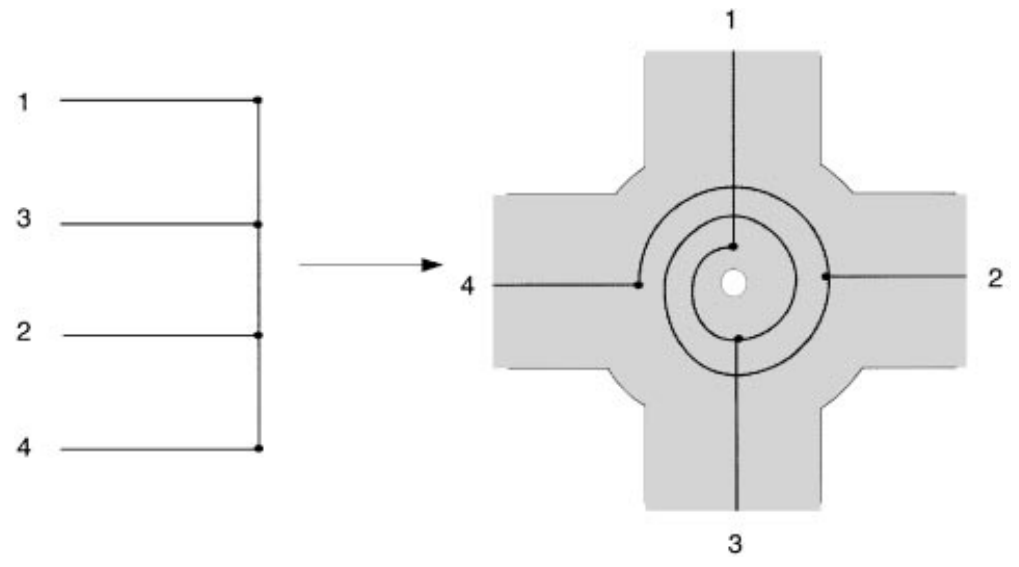

Fig. 1. A schematic picture of the map $\bar{f}_{\tau}$ of $X_{4}^{\tau}$ into $\widehat{M}_{4}$ where $\tau=(14)(2)(3)$. 
Let $C_{1}^{\prime}, \ldots, C_{k}^{\prime}$ be a family of consecutive, parallel, disjoint longitudinal curves on the torus $T=S^{1} \times S^{1}$. In this remark, we always traverse the meridinal factor of $T$ in the positively oriented direction. Let $Y$ be the 2-complex obtained from $T$ and $\{F(1), \ldots, F(k)\}$ by gluing $\partial F(j)$ to $C_{j}^{\prime}$. Then $\widehat{M}_{k}$ is a thickening of $Y$.

We can schematically describe $f_{\tau}$ by describing a map $\bar{f}_{\tau}: X_{k}^{\tau} \rightarrow Y$. Let $C_{0}^{\prime}$ be a longitudinal curve on $T$ between $C_{k}^{\prime}$ and $C_{1}^{\prime}$. We map $F(j)$ to $F(j)$, and hence $C_{j}$ to $C_{\tau(j)}^{\prime}$, but we map the region between $C_{j}$ and $C_{j+1}$ to the union of the region on $T$ between $C_{\tau(j)}^{\prime}$ and $C_{0}^{\prime}$ and the region between $C_{0}^{\prime}$ and $C_{\tau(j+1)}^{\prime}$. Notice that $\bar{f}_{\tau}$ "wraps" $A$ around $T$ at least $k-2$ times.

\section{Deformation spaces of Kleinian groups}

In this section we show that $C C\left(\pi_{1}\left(M_{k}\right)\right)$ has $(k-1)$ ! components and connected closure. We begin by describing a topological enumeration of the components of $C C\left(\pi_{1}\left(M_{k}\right)\right)$.

Consider the pair $\left(M^{\prime}, h^{\prime}\right)$ where $M^{\prime}$ is an oriented, compact, irreducible 3manifold and $h^{\prime}: M \rightarrow M^{\prime}$ is a homotopy equivalence. Two pairs $\left(M_{1}, h_{1}\right)$ and $\left(M_{2}, h_{2}\right)$ are equivalent if there exists a orientation-preserving homeomorphism $\phi: M_{1} \rightarrow M_{2}$ such that $\phi \circ h_{1}$ is homotopic to $h_{2}$. An equivalence class of such pairs is called a marked homeomorphism type of (oriented) 3-manifolds homotopy equivalent to $M$; the set of all such equivalence classes is denoted $\mathscr{A}(M)$.

Given a geometrically finite representation $\rho: \pi_{1}(M) \rightarrow \mathrm{PSL}_{2}(\mathbf{C})$, there is a homotopy equivalence $h_{\rho}: M \rightarrow N_{\rho}=\mathbf{H}^{3} / \rho\left(\pi_{1}\left(M_{k}\right)\right)$ such that $\left(h_{\rho}\right)_{*}$ is conjugate to $\rho$ and an orientation-preserving homeomorphism $\psi: N_{\rho} \rightarrow \operatorname{int}\left(M^{\prime}\right)$ from $N_{\rho}$ to the interior of some compact, oriented 3-manifold $M^{\prime}$. Hence, we may associate to $\rho$ the element $\left(M^{\prime}, \psi \circ h_{\rho}\right)$ of $\mathscr{A}(M)$. Marden's isomorphism theorem [17] asserts that two convex co-compact representations lie in the same component of $C C\left(\pi_{1}(M)\right)$ if and only if they give rise to the same element of $\mathscr{A}(M)$. Combining this with work of Ahlfors, Bers, Kra, Maskit and Thurston, one may prove (see [5]) that the components of $C C\left(\pi_{1}(M)\right)$ are in a one-to-one correspondence with elements of $\mathscr{A}(M)$. This topological enumeration should be considered as the analogue, via the Sullivan dictionary, of the combinatorial enumeration of the hyperbolic components of the Mandelbrot set (see [4]).

Theorem 3.1. Let $M_{k}$ be as in the previous section. Then $C C\left(\pi_{1}\left(M_{k}\right)\right)$ has $(k-1)$ ! components but has connected closure in $\mathscr{D}\left(\pi_{1}(M)\right)$.

Proof of 3.1. We will need a topological lemma which describes the elements of $\mathscr{A}\left(M_{k}\right)$. For each $\tau \in \mathbf{S}_{k}$, let $h_{\tau}: M_{k} \rightarrow M_{k}^{\tau}$ be a fixed homotopy equivalence which is the identity map restricted to the solid torus $V$. Let $j_{\tau}$ be a homotopy inverse for $h_{\tau}$. 
Lemma 3.2. If we let $\left\{\tau_{1}, \ldots, \tau_{n}\right\}$ denote a set of right coset representatives of the cyclic subgroup $\mathbf{Z}_{k}$ generated by $(123 \cdots n)$ in $\mathbf{S}_{k}$, then $\left\{\left(M_{k}^{\tau_{1}}, h_{\tau_{1}}\right), \ldots\right.$, $\left.\left(M_{k}^{\tau_{n}}, h_{\tau_{n}}\right)\right\}$ is a complete set of representatives for the elements of $\mathscr{A}\left(M_{k}\right)$.

Proof of 3.2. The proof of the lemma is a simple exercise in the JohannsonJaco-Shalen characteristic submanifold theory. We first note that, for $\tau \in$ $\mathbf{S}_{k}$, the characteristic submanifold $\Sigma\left(M_{k}^{\tau}\right)$ of $M_{k}^{\tau}$ consists of the $I$-bundles $\{B(1), \ldots, B(k)\}$ and a solid torus $V_{0}$ which is obtained from $V$ by removing a small regular neighborhood of each $A(j)$. (See Sect. 4 of [9].)

Johannson's theorem (Theorem 24.2 in [11]) asserts that, if $h: M_{k} \rightarrow M^{\prime}$ is a homotopy equivalence, then $h$ may be homotoped to a homotopy equivalence $\bar{h}$ such that $\bar{h}\left(\Sigma\left(M_{k}\right)\right)=\Sigma\left(M^{\prime}\right)$ and $\bar{h}$ is a homeomorphism of $M_{k}-\Sigma\left(M_{k}\right)$ to $M^{\prime}-\Sigma\left(M^{\prime}\right)$. Moreover (see Proposition 28.4 in [11]), we may assume that $\bar{h}$ is an orientation-preserving homeomorphism restricted to each $B(j)$. One may also check that the component $\Sigma_{0}$ of $\Sigma\left(M^{\prime}\right)$ which contains $h\left(V_{0}\right)$ is a solid torus and that the inclusion of each component of $\Sigma_{0} \cap \partial M^{\prime}$ in $\Sigma_{0}$ is a homotopy equivalence. It follows that $M^{\prime}$ is homeomorphic to $M_{k}^{\tau}$ for some $\tau$. (See also Proposition 4.3 in [9].) Therefore, every element of $\mathscr{A}\left(M_{k}\right)$ has a representative of the form $\left(M_{k}^{\tau}, h\right)$ for some $\tau$ and $h$.

We now consider a pair $\left(M_{k}^{\tau}, h\right)$ where $h: M_{k} \rightarrow M_{k}^{\tau}$ is a homotopy equivalence. Let $g$ be a generator of $\pi_{1}(V)$ sitting within $\pi_{1}\left(M_{k}\right)$. We may again use Johannson's theorem to homotope $h \circ j_{\tau}: M_{k}^{\tau} \rightarrow M_{k}^{\tau}$ to a homotopy equivalence $h \circ j_{\tau}$ such that $h \circ j_{\tau}(V) \subset V$ and $h \circ j_{\tau}$ restricts to an orientation-preserving homeomorphism of each $B(j)$.

There are now two possibilities. In the case that $h_{*}(g)$ is conjugate to $\left(h_{\tau}\right)_{*}(g)$, we may further assume that $h \circ j_{\tau}$ is the identity when restricted to $\partial_{0}(B(j))$, and hence that $h \circ j_{\tau}$ is homotopic to an orientation-preserving homeomorphism. Thus, in this case, $\left(M_{k}^{\tau}, h_{\tau}\right)$ is equivalent to $\left(M_{k}^{\tau}, h\right)$.

We now suppose that $h_{*}(g)$ is not conjugate to $\left(h_{\tau}\right)_{*}(g)$. Let $\sigma$ denote an odd element of the dihedral group $\mathbf{D}_{k} \subset \mathbf{S}_{k}$. There exists an orientationpreserving homeomorphism $\phi_{(\sigma, \tau)}$ from $M_{k}^{\tau}$ to $M_{k}^{\sigma \tau}$ obtained by "reflecting" about the core curve of $V$ (and reversing its orientation in the process). Note that $\left(M_{k}^{\tau}, h\right)$ is equivalent to $\left(M_{k}^{\sigma \tau}, \phi_{(\sigma, \tau)} \circ h\right)$ and $\left(\phi_{(\sigma, \tau)} \circ h\right)_{*}(g)$ is conjugate to $\left(h_{\sigma \tau}\right)_{*}(g)$. Hence, in this case, $\left(M_{k}^{\sigma \tau}, h_{\sigma \tau}\right)$ is equivalent to $\left(M_{k}^{\tau}, h\right)$.

It follows from the above arguments that every element of $\mathscr{A}\left(M_{k}\right)$ is equivalent to one of the form $\left(M_{k}^{\tau}, h_{\tau}\right)$ for some $\tau \in \mathbf{S}_{k}$. If $\tau$ and $\tau^{\prime}$ lie in the same right coset of $\mathbf{Z}_{k}$, we may construct an orientation-preserving homeomorphism from $M_{k}^{\tau}$ to $M_{k}^{\tau^{\prime}}$ by rotating $M_{k}^{\tau}$ along the core curve of $V$. Thus, $\left(M_{k}^{\tau^{\prime}}, h_{\tau^{\prime}}\right)$ is equivalent to $\left(M_{k}^{\tau}, h_{\tau}\right)$. It follows that every marked homeomorphism type in $\mathscr{A}\left(M_{k}\right)$ has a representative of the desired form. One completes the proof by using the same type of analysis to show that $\left(M_{k}^{\tau_{j}}, h_{\tau_{j}}\right)$ and $\left(M_{k}^{\tau_{i}}, h_{\tau_{i}}\right)$ are inequivalent if $i \neq j$.

It follows immediately from Lemma 3.2 that there are $(k-1)$ ! components of $C C\left(\pi_{1}\left(M_{k}\right)\right)$. 
Given $\tau$, let $\left\{\rho_{n}\right\}$ be as in Theorem 2.1. Theorem 2.2 guarantees that there exists $\phi_{n}: \operatorname{int}\left(\widehat{M}_{k}(1, n)\right) \rightarrow \mathbf{H}^{3} / \rho_{n}\left(\pi_{1}\left(M_{k}\right)\right)$ such that $\left(\phi_{n} \circ i_{n}\right)_{*}$ is conjugate to $\beta_{n} \circ \phi_{*}$. Hence, $\rho_{n}$ lies in the component of $C C\left(\pi_{1}(M)\right)$ associated to the element $\left(\widehat{M}_{k}(1, n), i_{n} \circ f_{\tau} \circ h_{\tau}\right)$ of $\mathscr{A}(M)$. We note that $\left(f_{\tau} \circ h_{\tau}\right)_{*}(g)$ is homotopic to $f_{*}(g)$, so the analysis in the proof of Lemma 3.2 implies that $\left(\widehat{M}_{k}(1, n), i_{n} \circ f_{\tau} \circ h_{\tau}\right)$ is equivalent to $\left(\widehat{M}_{k}(1, n), i_{n} \circ f\right)$. Since there is an orientation-preserving homeomorphism from $M_{k}$ to $\widehat{M}_{k}(1, n)$ which is homotopic to $i_{n} \circ f$, we see that $\left(\widehat{M}_{k}(1, n), i_{n} \circ f\right)$ is equivalent to $\left(M_{k}\right.$, id $)$ where id : $M_{k} \rightarrow M_{k}$ is the identity map. Therefore, every $\rho_{n}$ lies in the component of $C C\left(\pi_{1}\left(M_{k}\right)\right)$ associated to $\left(M_{k}\right.$, id), and so $\rho$ lies in the boundary of the component of $C C\left(\pi_{1}\left(M_{k}\right)\right)$ associated to $\left(M_{k}\right.$, id $)$.

One may similarly check that $\rho$ is associated to the element $\left(M_{k}^{\tau}, h_{\tau}\right)$ of $\mathscr{A}(M)$. It then follows from Corollary 6 of [23] that $\rho$ also lies in the boundary of the component of $C C\left(\pi_{1}\left(M_{k}\right)\right)$ corresponding to $\left(M_{k}^{\tau}, h_{\tau}\right)$. (One may also use Theorem 2.2 to construct a sequence $\left\{\rho_{n}^{\tau}\right\}$ in the component of $C C\left(\pi_{1}\left(M_{k}\right)\right)$ corresponding to $\left(M_{k}^{\tau}, h_{\tau}\right)$ which converges to $\rho$.) As $\tau$ was arbitrary, we see that $C C\left(\pi_{1}\left(M_{k}\right)\right)$ has connected closure.

Remark 3. Notice that the technique of proof may also be used to show that the closures of any two components of $C C\left(\pi_{1}\left(M_{k}\right)\right)$ intersect.

Remark 4. One may use work of [1] and [5] to show that there exist manifolds $M$ such that $C C\left(\pi_{1}(M)\right)$ has arbitrarily many components, all of whose closures are distinct. For example, the components of $C C\left(\pi_{1}(M)\right)$ will have disjoint closures whenever $M$ has incompressible boundary and every component of its characteristic submanifold is a solid torus which intersects the interior of the manifold in a collection of annuli whose fundamental groups are not maximal cyclic subgroups of $\pi_{1}(M)$. There also exist manifolds $M$ with incompressible boundary such that $\mathscr{D}\left(\pi_{1}(M)\right)$ has infinitely many components. The above results, together with a more general discussion of the connectivity of deformation spaces, will be contained in [2].

Acknowledgements. The authors would like to thank Steve Kerckhoff and Curt McMullen for helpful and enjoyable discussions. We would especially like to thank Yair Minsky for his comments on early versions of this manuscript and for drawing the picture.

\section{References}

1. Anderson, J.W., Canary, R.D.: Cores of hyperbolic 3-manifolds and limits of Kleinian groups, Amer. J. Math. (to appear)

2. Anderson, J.W., Canary, R.D., McCullough, D.: Shuffling homotopy equivalences and deformation spaces of Kleinian groups, in preparation

3. Bonahon, F., Otal, J.P.: Variétés hyperboliques à géodésiques arbitrairement courtes, Bull. L.M.S. 20, 255-261 (1988)

4. Branner, B.: Puzzles and para-puzzles of quadratic and cubic polynomials, in Complex Dynamical Systems, Proc. Symp. in Appl. Math. 49, 31-69 (1994)

5. Canary, R.D., McCullough, D.: Homotopy equivalences of 3-manifolds and deformation theory of Kleinian groups, in preparation 
6. Canary, R.D., Minsky, Y.N.: On limits of tame hyperbolic 3-manifolds, J. Diff. Geom., 43, 1-41 (1996)

7. Chuckrow, V.: On Schottky groups with applications to Kleinian groups, Ann. Math. 88, 47-61 (1968)

8. Comar, T.D.: Hyperbolic Dehn surgery and convergence of Kleinian groups, Ph.D. Thesis, University of Michigan, 1996

9. Culler, M., Shalen, P.B.: Volumes of hyperbolic Haken manifolds I, Invent. math. 118, 285-329 (1994)

10. Jaco, W., Shalen, P.B.: Seifert fibered spaces in 3-manifolds, Memoirs of the American Mathematical Society 220 (1979)

11. Johannson, K.: Homotopy equivalences of 3-manifolds with boundaries, Lecture notes in Mathematics, vol. 761, Springer-Verlag, 1979

12. Jørgensen, T.: On cyclic groups of Mobius transformations, Math. Scand. 33, 250-260 (1973)

13. Jørgensen, T.: On discrete groups of Möbius transformations, Amer. J. Math. 98, 739$749(1976)$

14. Jørgensen, T., Klein, P.: Algebraic convergence of finitely generated Kleinian groups, Quart. J. Math. Oxford 33, 325-332 (1982)

15. Jørgensen, T., Marden, A.: Algebraic and geometric convergence of Kleinian groups, Math. Scand. 66, 47-72 (1990)

16. Kerckhoff, S.P., Thurston, W.P.: Non-continuity of the action of the mapping class group at Bers' boundary of Teichmüller space, Invent. math. 100, 25-47 (1990)

17. Marden, A.: The geometry of finitely generated Kleinian groups, Ann. of Math. 99, $383-462(1974)$

18. Marden, A.: Geometric relations between homeomorphic Riemann surfaces, Bull. A.M.S. 3, 1001-1017 (1980)

19. Maskit, B.: Kleinian groups, Springer-Verlag, 1988

20. Maskit, B.: Panelled web groups, in Kleinian groups and related topics, ed. by D.M. Gallo and R.M. Porter, Lecture Notes in Mathematics, vol. 971, Springer-Verlag, 1983

21. McCullough, D., Miller, A., Swarup, G.A.: Uniqueness of cores of non-compact 3-manifolds, J. London Math. Soc. 32, 548-556 (1985)

22. Morgan, J.W.: On Thurston's uniformization theorem for three-dimensional manifolds, in The Smith Conjecture, ed. by J. Morgan and H. Bass, Academic Press, 37-125 (1984)

23. Ohshika, K.: Ending laminations and boundaries for deformation spaces of Kleinian groups, J. London Math. Soc. 42, 111-121 (1990)

24. Ohshika, K.: Geometric behaviour of Kleinian groups on boundaries for deformation spaces, Quart. J. Math. Oxford 43, 97-111 (1992)

25. Ohshika, K.: Kleinian groups which are limits of geometrically finite groups, preprint

26. Sullivan, D.P.: Quasiconformal homeomorphisms and dynamics II: Structural stability implies hyperbolicity of Kleinian groups, Acta Math. 155, 243-260 (1985)

27. Thurston, W.P.: The geometry and topology of 3-manifolds, lecture notes, Princeton University

28. Thurston, W.P.: Hyperbolic structures on 3-manifolds II: Surface groups and 3manifolds which fiber over the circle (preprint) 